\title{
Transducer based on surface plasmon resonance with thermal modification of metal layer properties
}

\author{
K.V. Kostiukevych \\ V. Lashkaryov Institute of Semiconductor Physics, NAS of Ukraine, 41, prospect Nauky, 03680 Kyiv, Ukraine \\ E-mail:biosen@isp.kiev.ua
}

\begin{abstract}
With the purpose to improve such service characteristics of transducers on the basis of the surface plasmon resonance (SPR) as the sensitivity and stability, we have analyzed the influences of a structure and a relief of the surface of polycrystalline gold films, which are determined by technological conditions of their production and by the low-temperature annealing, on their optical characteristics, namely, the coefficients of refraction, extinction, and light scattering. It is shown that the mechanism of enhancement of the sensitivity of an SPR-based transducer consists in a decrease in the coefficient of extinction of a metal film, as the annealing temperature increases. At the optimum annealing temperature $\left(120^{\circ} \mathrm{C}\right)$, we observed the smoothing of a small-scale roughness of the gold film surface, which decreases the scattering of plasmons, favors the defectless formation of protective nano-sized layers, and enhances the sensitivity and stability of the operation of SPR-based transducers.
\end{abstract}

Keywords: surface plasmon resonance, gold films, low-temperature annealing, microrelief of the surface, optical constants, service characteristics of an SPR-based transducer.

Manuscript received 20.04.16; revised version received 05.07.16; accepted for publication 13.09.16; published online 04.10.16.

\section{Introduction}

In several last decades, we observe the growing interest in tiny, low-cost, and sensitive optical sensors used in the direct studies of a molecular interaction in the real time scale without marks and applied to such fields as the monitoring of the environment and industrial wastes, estimation of the quality of products, discovery of new drugs, clinical diagnostics, etc. [1,2]. In such sensors, one of the interacting molecules is immobilized on the working surface of a sensor, which forms a sensitive element. The attachment of an interacting partner is controlled with the help of the measurement of changes in the optical density on the sensor surface. The method of surface plasmon resonance (SPR) is the most developed sensor technology widely used for the detection of chemical and biological substances [3-5]. However, the devices on the basis of SPR possess else such drawbacks as the bounded sensitivity, low productivity, and high cost $[6,7]$.

We recall that the surface plasmon polaritons (SPPs) are electromagnetic waves of the TM-type, which are localized on the interface of two media. One of the media is surface-active, i.e., it has a negative dielectric 
permeability in some range of frequencies [8]. In the case of a plane interface, the wave vector of an SPP is larger than the projection of the wave vector of an exciting electromagnetic wave on the interface of two media. Therefore, in order to excite an SPP, it is necessary to satisfy the condition of phase synchronism (the law of conservation of a momentum in the system). Among the most spread means to ensure the phase synchronism, we mention the method of weakened prism-based total reflection (in the Kretschmann [9] and Otto [10] configurations) and the method of diffraction grating. At the excitation of an SPP, the electromagnetic field intensity is amplified in a resonance way on the interface and decays rapidly on both sides from it. If an SPP is excited in a metal film, the surface wave is called a surface plasmon (SP). The excitation of an SP is characterized by the phenomenon of surface plasmon resonance and is registered in the shape of a SPR curve with the help of such methods of measurement of the coefficient of reflection from the metal/dielectric interface as the sampling of the incidence angle at a fixed wavelength of light, scanning of a light wavelength at a fixed incidence angle, or the combination of both methods [11]. The conditions of propagation of SPs are determined by the optical properties of contacting layers of a metal and a dielectric (analyzed substance) and, hence, are sensitive to their insignificant changes. Thus, the sensitivity and stability of the operation of transducers on the basis of SPR will depend to a significant extent on the properties of a metal film.

The problem of development of sensor devices depends on the principle of measurements of SPR and requires a proper choice of the working wavelength [12], as well as the type and the thickness of a metal film [13], which will allow one to optimize the sensitivity of a device. Such metals as silver $(\mathrm{Ag})<$ gold $(\mathrm{Au})<$ copper $(\mathrm{Cu})<$ aluminum $(\mathrm{Al})$ reveal the narrowest resonance curves as compared with other metals in the visible and near-IR parts of the electromagnetic spectrum and, hence, are the most suitable for the use in sensor devices. Gold is preferred due to a high chemical stability under the action of the atmosphere, though this metal does not reveal the sharpest resonance and has no resonance at wavelengths less than $0.5 \mu \mathrm{m}$. The use of bimetallic layers (e.g., $\mathrm{Ag} / \mathrm{Au}$ ) [14] solves the problem of enhancement of the sensitivity under the conservation of inertness of the working surface of an SPR-based transducer, but complicates its fabrication. The problem of low adhesion of noble metals to dielectric substrates [15] can be solved with the use of intermediate layers of such metals as tungsten, molybdenum, and chromium. The highest stability to mechanical actions and chemical and thermal stabilities are manifested by chromium, which is mainly used to increase the cohesion of a gold film with a transparent dielectric substrate.

The studies of the properties of metal films testify to the essential dependence of their structure and physico-chemical and optical properties on the production technology [16,17]. The relief of a surface is the strongest factor distorting the patterns of the absorption and the scattering of light by the mechanism of inhomogeneous field. The surface roughness of a metal affects essentially the propagation of surface plasmons, by causing their damping, a decrease in the phase velocity, and a change in the dispersion relation due to the single or multiple scattering [18-20]. On the other hand, the surface properties of films determine the mechanisms of adsorption and formation of nano-sized molecular layers on the working surface of an SPRbased transducer [21,22]. The real metal films have always a number of structural imperfections (defects, pores, interfaces of crystallites, admixtures, etc.), which cause fluctuations of the dielectric permeability, define the character of the absorption and the scattering of light, and lead to a variation in parameters of a film in the course of the time ("ageing") or due to the interaction with the medium under study [23]. This violates the stability and the reproducibility of working characteristics of an SPRbased transducer. The roughness of the surface and the inhomogeneity of the film bulk will affect the SPR angle and the absorption band shape and cause a distortion of the SPR curve, a decrease in the sensitivity of a sensor device to the adsorbed molecules, and errors in the calculations of optical parameters of a metal film (deterioration of the discrepancy of an approximation). Therefore, it is obvious that the use of thin-film metal coatings in the sensor instrument-making industry requires their detailed characterization from the viewpoint of the technology-properties connection.

\section{Experimental results and analysis}

By executing the studies, we used the method of SPR spectroscopy with the prism-based excitation by the Kretschmann scheme in a configuration with scanning of the incidence angle with a mechanical sweeping, which ensures the measurement of the complete resonance curve in the limits of 18 degrees in air (12 degrees in glass). To attain the most efficient operation of a transducer, the thickness of a metal film is chosen from the condition of a minimum reflection at the minimum of the resonance $\left(R_{\min }\right)$, which can be determined on the basis of numerical calculations $[12,13]$. In this case, we assume that the metal film has a homogeneous internal structure and a plane-parallel surface. It is clear that the real situation differs essentially from the presented ideal model. Therefore, the optimum thickness of a gold film and the influence of an adhesive chromium sublayer were found in a practical way.

\subsection{The technology of fabrication of an SPR-based transducer}

The sensitive element of an SPR-based transducer was fabricated with the use of the method of thermal evaporation in vacuum by an optimum technology. For 
the execution of studies, we chose polished quartz $\left(n_{S}=1.475\right)$ substrates with a low mean-square roughness of the surface $(c a .1 .1 \mathrm{~nm})$. The specimens were purified in mechanical and chemically ways (holding in a chromate mixture containing $10 \mathrm{~g}$ of $\mathrm{K}_{2} \mathrm{Cr}_{2} \mathrm{O}_{7}$ in $350 \mathrm{ml}$ of $\mathrm{H}_{2} \mathrm{SO}_{4}$, with the subsequent washing in a large amount of distilled water with the use of an ultrasound bath) and subjected to a finishing processing in a glow discharge immediately before the sputtering. The gold films $(30 \div 60) \mathrm{nm}$ in thickness were sputtered by the method of thermal evaporation in vacuum (VUP-4, a residual vapor pressure of $4 \cdot 10^{-4} \mathrm{~Pa}$, a sputtering rate of $40-50 \AA / \mathrm{s}$ ) on a chromium layer (thickness $c a .1 \mathrm{~nm}$ ) improving the adhesion without heating of a substrate and were thermally annealed in air for $30 \mathrm{~min}$ in the temperature interval $80 \div 200^{\circ} \mathrm{C}$.

We measured the SPR curves of the specimens in air with the use of a goniometer (G5M), glass prism (angle of $45^{\circ}, n_{P}=1.52$ ), glycerol as the immersion liquid $\left(n_{G}=1.45\right)$, and a He-Ne laser $(\lambda=632.8 \mathrm{~nm})$ as a light source. Before studying the dependence of the working characteristics of SPR-based transducers on the modes of thermal annealing, we analyzed the influence of the metal layer thickness $d_{M}$ (Fig. 1) and the presence of the adhesive Cr layer (Fig. 2) on the resonance curves.

It is seen from Fig. 1 that a decrease or an increase in $d_{M}$ renders a significant influence on the shape of SPR curves: if $d_{M}$ exceeds the optimum value, then the value of reflection at the minimum of the resonance $R_{\min }$ is increased, if $d_{M}$ is less than the optimum value, the halfwidth of the resonance curve $\Delta R$ is increased. It is worth to note the fact that the influence of the annealing temperature depends on the thickness of films. So, if $d_{M}$ exceeds the optimum value, then the annealing shifts the SPR curve to larger angles, if $d_{M}$ is less than the optimum value, then the curve is shifted to smaller angles. For the optimum thickness of the gold film $(\approx$ $45 \mathrm{~nm}$ ), no shift is observed. Therefore, all subsequent studies are carried out for films $(40 \pm 5) \mathrm{nm}$ in thickness.

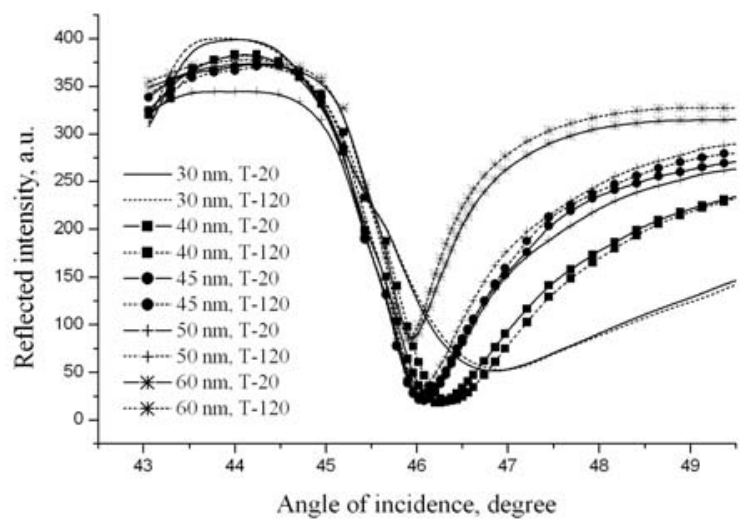

Fig. 1. SPR curves (air) for gold films $(30 \div 60) \mathrm{nm}$ in thickness sputtered on quartz substrates at the temperature $T_{S}=20^{\circ} \mathrm{C}$ and annealed at $T_{A}=120^{\circ} \mathrm{C}$.

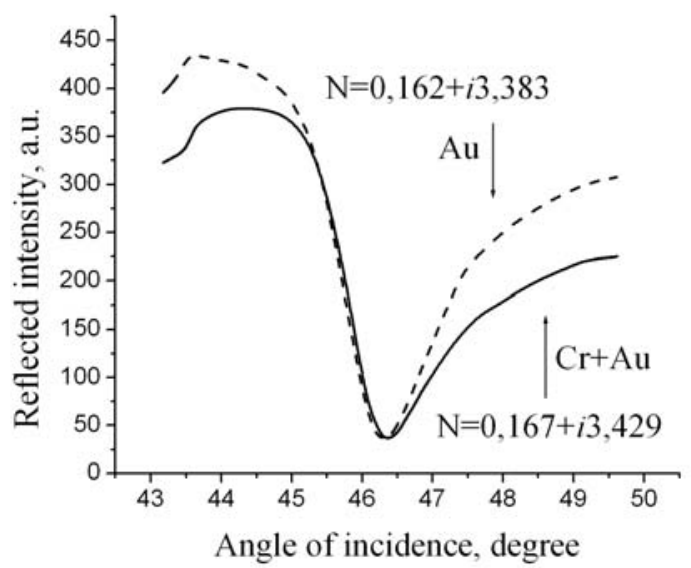

Fig. 2. SPR curves (air) for gold films $\left(d_{M} \approx 45 \mathrm{~nm}\right)$ with an adhesive chromium sublayer $\left(d_{C r} \approx 1 \mathrm{~nm}\right)$ and without it, which were sputtered on quartz substrates at the temperature $T_{S}=$ $20^{\circ} \mathrm{C}$.

Figure 2 shows that the curves slightly differ from one another at the minimum (shift is $\approx 0.06$ degr.). At the same time, the SPR curve for the structure $\mathrm{Cr} / \mathrm{Au}$ has a larger half-width (difference is 0.5 degr.) and the small value of a reflected signal at the transition to the distorted total internal reflection region and at larger angles (on the shoulders of a curve). In this case, the system $\mathrm{Cr} / \mathrm{Au}$ can be considered as a single metal layer with effective optical constants [24]. On the basis of the obtained experimental data for the coatings under study within the one-layer optical model, we calculated the values of a complex-valued coefficient of refraction by the method of fitting of the theoretical SPR curves to the experimental ones [25]. For a gold film, $N_{A u}=0.162+$ $i$ 3.383, whereas $N_{C r+A u}=0.167+i 3.429$ for a gold film with $\mathrm{Cr}$ sublayer.

\subsection{Morphological and topographical peculiarities of the thin-film structure Cr/Au under the action of a low- temperature annealing}

The crystal structure of specimens was studied by the method of diffraction of the X-ray characteristic emission of copper [26]. The angular distribution of maxima testifies that the films have a polycrystalline structure with the dominant orientation of crystallites in the direction $<011>$ and indicate the presence of a quasiamorphous phase of gold with a characteristic size of grains of $5-10 \AA$. As the annealing temperature increases, the texture of films becomes more pronounced, and the size of grains increases.

To describe a reconstruction of the surfaces of gold films under the action of a thermal annealing, we used the complex analysis of the characteristics of images of the surface, which were obtained with the help of the method of atomic-force microscopy (AFM) [27] on a microscope (Nanoscope IIIa Dimension 3000, Digital 

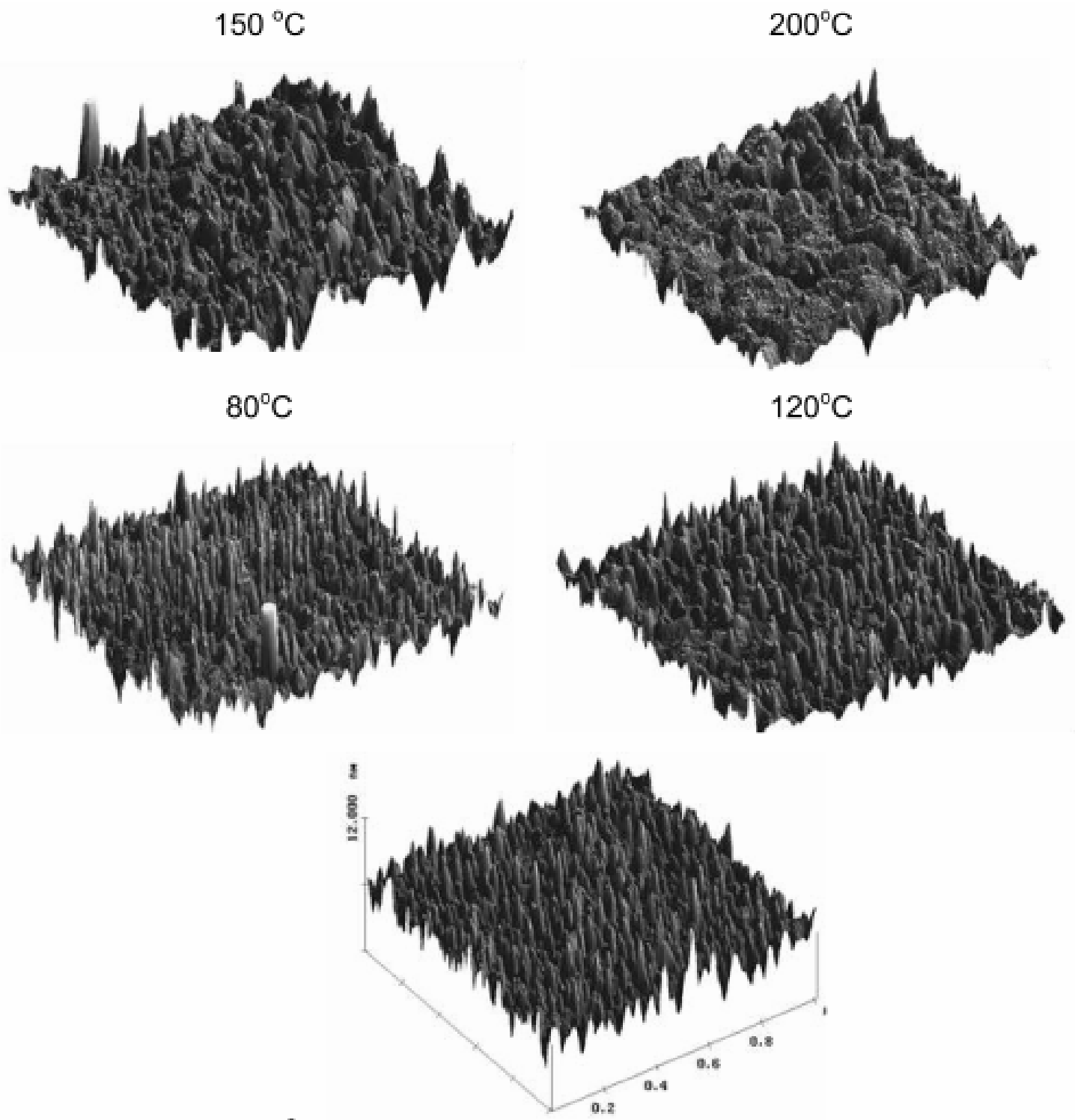

$20^{\circ} \mathrm{C}$

Fig. 3. AFM image of the gold film surface without the annealing (below) and annealed (above) in air for 30 min at 80, 120, 150, and $200^{\circ} \mathrm{C}(1 \times 1 \mu \mathrm{m}$, mode of tapping contact, a microscope Nanoscope IIIa, Digital Instrument, Santa-Barbara, CA).

Instrument, Santa-Barbara, CA) equipped with a $80-\mu \mathrm{m}$ piezoscanner. The measurements were carried out in air at room temperature on an elastic console element with the elasticity coefficient of $0.01 \div 0.6 \mathrm{~N} / \mathrm{m}$, by using the probes $\left(\mathrm{Si}_{3} \mathrm{~N}_{4}\right)$ with a nominal curvature radius of the tip $\sim 10 \mathrm{~nm}$. The three-dimensional images of the surfaces of specimens under study (Fig. 3) were obtained in the mode of periodic contact (tapping mode) with a scanning frequency of about $1 \mathrm{~Hz}$.

The histograms of the distribution over sizes of grains in the substrate plane are obtained on the basis of the program Scion Image aimed at the numerical analysis of AFM images. In the indicated approach, all objects of the surface (individual crystallites, their clusters, etc.) were approximated by an ellipse, whose major axis was considered as the grain diameter. We found that the most probable diameter of grains for the films sputtered at $20^{\circ} \mathrm{C}$ and $T_{A}=80^{\circ} \mathrm{C}$ is in the interval from 20 to $40 \mathrm{~nm}$. For $T_{A}=120^{\circ} \mathrm{C}$, we observe an insignificant shift of the histogram maximum to $50 \mathrm{~nm}$. The shifts are larger for $T_{A}=150^{\circ} \mathrm{C}(80 \mathrm{~nm})$ and $T_{A}=200^{\circ} \mathrm{C}(150 \mathrm{~nm})$ and are accompanied by its spreading.

The mean square roughness of a surface (MSR) is a statistical parameter characterizing a variation in the 
height of a rough surface in the direction perpendicular to the substrate without regard for the spatial ratio [28]. The value of MSR is defined as the square root of the mean distance from points of the surface to the average level of the surface and is equal to $0.93 \mathrm{~nm}$ for the films sputtered at $20^{\circ} \mathrm{C}$. It irregularly varies with increase in the annealing temperature: it increases firstly to $1.1 \mathrm{~nm}$ at $80{ }^{\circ} \mathrm{C}$ and then decreases down to $0.84 \mathrm{~nm}$ at $120^{\circ} \mathrm{C}$. At $150{ }^{\circ} \mathrm{C}$, the MSR is $0.95 \mathrm{~nm}$, and it decreases down to $0.68 \mathrm{~nm}$ at $200^{\circ} \mathrm{C}$. The nonmonotonic character of variation in the MSR under the action of a lowtemperature annealing of thin polycrystalline films is outside the limits of experimental errors and agrees with available literature data [29].

The power spectral density (PSD) function is the space-frequency spectrum of a rough surface [30], is measured in reciprocal-length units in parallel to the substrate plane, and gives information about the spatial frequencies causing the scattering of light. The sense of a PSD function can be clarified, if we cut the real surface with a plane perpendicular to the median plane of the surface. Such cutting gives a one-dimensional profile which can be presented by a superposition of sinusoidal waves with different amplitudes, periods, and phases, i.e., a Fourier series. The coefficients $F(x)$ of different frequency components in the Fourier series determine the PSD function. To be more exact, the PSD function is the square of the Fourier-transform of the initial profile of a rough surface $u(x)$ :

$F(x)=\left|\int_{\infty}^{\infty} u(x) \cdot e^{i \frac{2 \pi x}{\lambda} d x}\right|^{2}$.

It is obvious that the value of $F(\lambda)$ characterize the number of structures with the given wavelength and can be considered as a share of the area occupied by them.

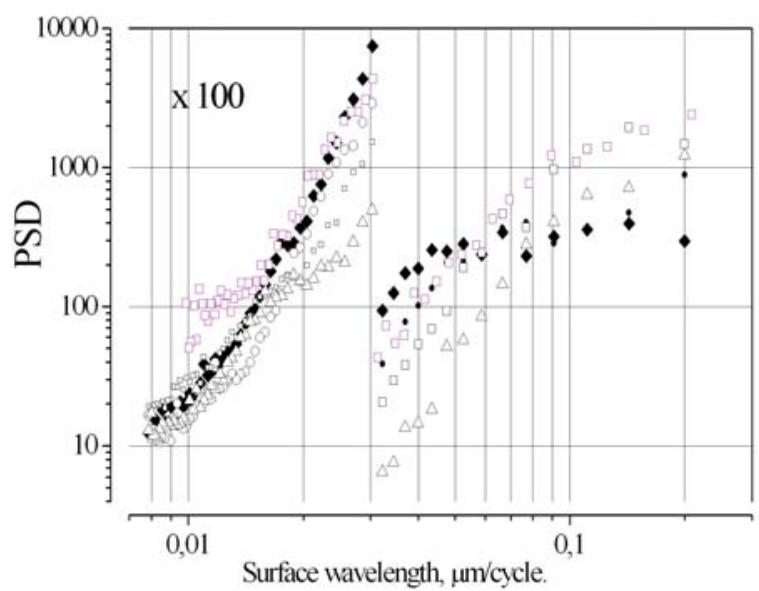

Fig. 4. Dependence of the power spectral density functions on the spatial wavelength on the surface of gold films produced at the substrate temperature $T_{S}=20^{\circ} \mathrm{C}$ and modified by the thermal annealing at $T_{A}=80,120,150$, and $200^{\circ} \mathrm{C}$.
The dependence of $F(\lambda)$ for the specimens under study (Fig. 4) has the form of an $s$-like curve in logarithmic coordinates, whose slope $d \lg F / d \lg \lambda$ is approximately 4,6 , and 2 for the sections with $\lambda<10 \mathrm{~nm}, 10<\lambda<60 \mathrm{~nm}$, and $\lambda>60 \mathrm{~nm}$, respectively. This testifies to the presence of some collection of local structures with sizes from several to hundreds of nanometers on the surface. It is seen that the total view of the curves of PSD functions is independent of the annealing temperature. But each of the indicated sections has some peculiarities. In the first section, all curves manifest the same dependence on the surface wavelength and are responsible, probably, for the properties of a small-height relief on the tops and in dents of crystallites. The following range of the lengths of surface wave characterizes the peculiarities of the height distribution in the region of sizes of individual crystallites and dents. In this region, the noticeable variations in the PSD function spectrum are observed at temperatures of 150 and $200^{\circ} \mathrm{C}$. The region above $60 \mathrm{~nm}$ (the inflection point of functions) represents the statistical distribution of the groups of crystallites on the surface.

Thus, we may conclude that the size of grains of the dominant crystallites varies insignificantly at a low annealing temperature, whereas the microrelief of the surface for the films annealed at temperatures of $150^{\circ} \mathrm{C}$ and $200^{\circ} \mathrm{C}$ differs from the rest ones and can be characterized by a more ordered structure of the surface. In addition, the statistical properties of the surface relief of the films under study can be divided into two groups: with higher values of $F(\lambda)$ (annealed at $80^{\circ} \mathrm{C}$ and $150^{\circ} \mathrm{C}$ ) and lower values of $F(\lambda)$ (without annealing and with annealing at $120^{\circ} \mathrm{C}$ and $200^{\circ} \mathrm{C}$ ), which correlates with the values of MSR.

The executed analysis of AFM images reflects only statistical (with the use of scalar and vector quantities) peculiarities of the studied rough surfaces, whereas the physical mechanism of their reconstruction under the action of a thermal annealing remains outside of such approach. The main reason for such situation consists in the loss of the information about a local structure, which is due to the statistical averaging not considering the dependence of properties of the surface on its height.

The distribution function over heights is a histogram showing a share of the surface height, which falls in the interval between the given height and the height increased by an infinitely small increment. In the given analysis, the main parameters of the estimate of properties of the surface are the asymmetry and the excess. The asymmetry is the measure of symmetry of the profile relative to the mean level of the surface (zero level). The excess is the measure of sharpness of the distribution function over heights and characterizes the localization of irregularities relative to the mean level of the surface. In Fig. 5, we present the histograms of the distribution function over heights for the annealed films relative to untreated specimens. It is seen that the functions have the shape of a bell (close to the Gauss one) and are centered near the zero level. 
The annealing at $80^{\circ} \mathrm{C}$ results only in a redistribution of the function for $H<0$, i.e., below the zero level. Namely, the number of irregularities, which exist at low values of $H<-1.2$, increases for the films annealed at $80^{\circ} \mathrm{C}$ relative to nonannealed specimens. The inverse effect is observed for the function in the interval $1.2 \mathrm{~nm}<H<0 \mathrm{~nm}$, which indicates some smoothing of valleys of the rough surface due to, probably, a decrease in the number of particles of gold, which were initially localized on the tops of small-sized irregularities inside those valleys. The coincidence of the right-hand sides of two height distribution functions indicates that the fracture faces localized on the tops of crystallites remain invariable.

The comparison of the histograms of specimens annealed at $120^{\circ} \mathrm{C}$ with untreated ones testifies to the surface transformation to the side of increasing the function near the zero plane $(H= \pm 0.5 \mathrm{~nm})$ and to a simultaneous decrease at low and high values of $\mathrm{H}$. These data can characterize the small-scale smoothing over the whole relief in valleys and on tops. Since the form of histograms is not essentially changed after this reconstruction, we may assume that the long-scale profile of the surface remains invariable.

The height distribution function for specimens annealed at $150^{\circ} \mathrm{C}$ is not essentially changed relative to specimens annealed at $120^{\circ} \mathrm{C}$. However, we can distinguish small changes near valleys and the onset of a growth near the zero level. Therefore, we can assume that the formation of new topographical structures starts at $150{ }^{\circ} \mathrm{C}$. The further increase in the annealing temperature to $200{ }^{\circ} \mathrm{C}$ gives a significant narrowing of a histogram, which testifies to the essential change in a structure of the interface.

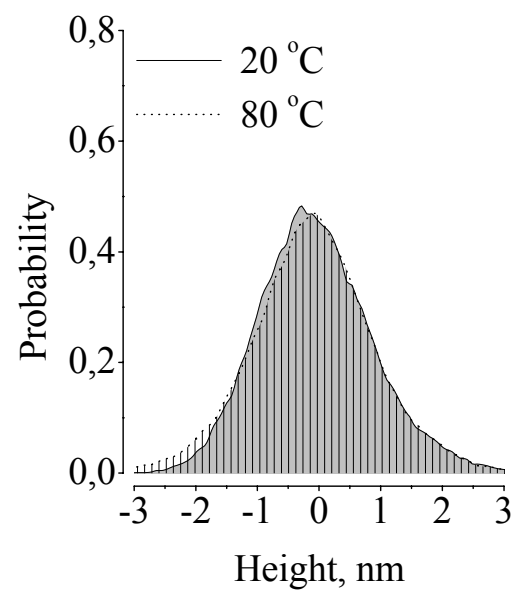

$a$

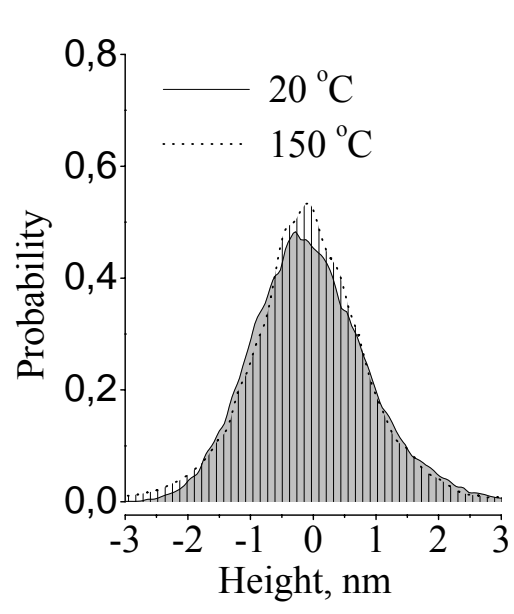

c

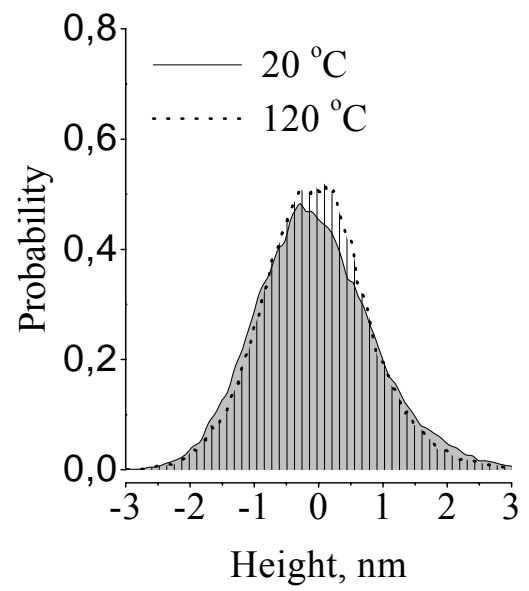

$b$

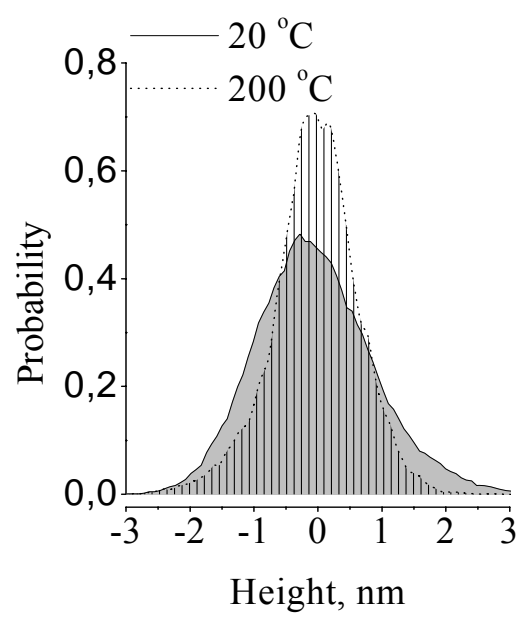

$d$

Fig. 5. Probability of the distribution function over heights relative to the zero plane of the surface for annealed gold films at the annealing temperatures $\mathrm{a}-80^{\circ} \mathrm{C}, \mathrm{b}-120^{\circ} \mathrm{C}, \mathrm{c}-150^{\circ} \mathrm{C}, \mathrm{d}-200^{\circ} \mathrm{C}$ as compared with specimens without the annealing. 
Thus, the complex analysis of the characteristics of AFM images allows us to determine the maximum variation in a relief of the surface $(2.5 \mathrm{~nm})$ and the diameter of grains in the substrate plane $(20 \div 200 \mathrm{~nm})$, as well as to describe the process of reconstruction of the surface microrelief of thin gold films as result of the low-temperature annealing. In the temperature interval $20-80^{\circ} \mathrm{C}$, the surface is characterized by the onset of the melting of small-sized irregularities, which are localized on the bottoms of valleys of the surface. These results enhance the values of mean square roughness of the surface and the PSD. For temperature interval $80-130^{\circ} \mathrm{C}$, the reconstruction of small-scale inhomogeneous fracture faces occurs already along the whole surface of crystallites and causes the smoothing of the surface of each crystallite. The shape of a surface relief changes from "rough" to "plane" only on a small scale, whereas the large-scale fracture faces are conserved. We may assume that, at a temperature of $130^{\circ} \mathrm{C}$, the reconstruction of the surface valleys is completed. The increase in the annealing temperature to $150^{\circ} \mathrm{C}$ causes a transformation of the tops of crystallites. Moreover, the results of such process are a smoothing of the top regions of crystallites and an increase in their sizes. However, for each crystallite, the temperature of such process depends on the surface tension and the surface curvature. The further increase in the temperature $\left(200^{\circ} \mathrm{C}\right)$ leads to the formation of plane domains on the tops of crystallites. In thin films, we observe a phase transition, which is caused by the processes of recrystallization and leads to the formation of crystallites with larger sizes and to the smoothing of a surface relief.

\subsection{Optical characteristics of the thin-film structure $\mathrm{Cr} / \mathrm{Au}$ under the action of a low-temperature annealing}

A specific feature of the method of surface plasmon resonance is the detection of changes in the coefficient of refraction $\Delta n$ in a layer with thickness $d$, which happens on the metallic surface of a transducer due to the adsorption of molecules or the running of the molecular interaction reaction. Such change in the physical parameters of the medium under study causes a shift of the SPR angle $\left(\Delta \theta_{S P R}\right)$. Hence, the efficiency of an SPR-based sensor can be characterized by the transformation constant $K$

$$
K=\frac{\Delta \theta_{\Pi \Pi P}}{\Delta n \cdot d},
$$

which determined the degree of transformation of the information in the process of biochemical interaction into the corresponding output signal of a device.

To estimate the influence of optical constants of a metal film (the coefficient of refraction of a metal $n_{M}$ and the coefficient of extinction of a metal $k_{M}$ ) on the transformation constant $K$, we carried out a numerical modeling of the response of an SPR-based transducer on the basis of the Fresnel formulas and the method of Jones matrices within the one-layer model of a gold coating under condition of its optimum thickness $\left(d_{M}=45 \mathrm{~nm}\right)$ for an adsorbed layer of biological molecules $\left(n_{D}=1.5\right)$ with the thickness $d_{D}=10 \mathrm{~nm}$ in water $\left(n_{L}=1.33\right)$.

The theoretical calculations showed that a change in the coefficient of refraction of a metal coating practically has no influence on the transformation constant $K$. At the same time, a variation of the coefficient of extinction leads to the exponential dependence of a shift of the resonance angle $\Delta \theta_{S P R}$, which is demonstrated by Fig. 6 . This dependence testifies that the less the modulus of the coefficient of extinction of a metal film, the larger the transformation constant $K$. Hence, in order to increase the sensitivity of the method, it is necessary to decrease the coefficient of extinction of a metal film. However, in this case, the resonance curve will spread and shift to larger angles. Since the determination of a minimum of the SPR curve is executed on the basis of a polynomial approximation of experimental SPR dependences in a vicinity of the minimum $\left(\theta_{S P R} \pm 0.5^{\circ}\right)$, the spreading of the curve will render no significant influence on the accuracy of its determination. A shift of the SPR curves to larger angles can be easily compensated by the choice of a corresponding angle of the prism.

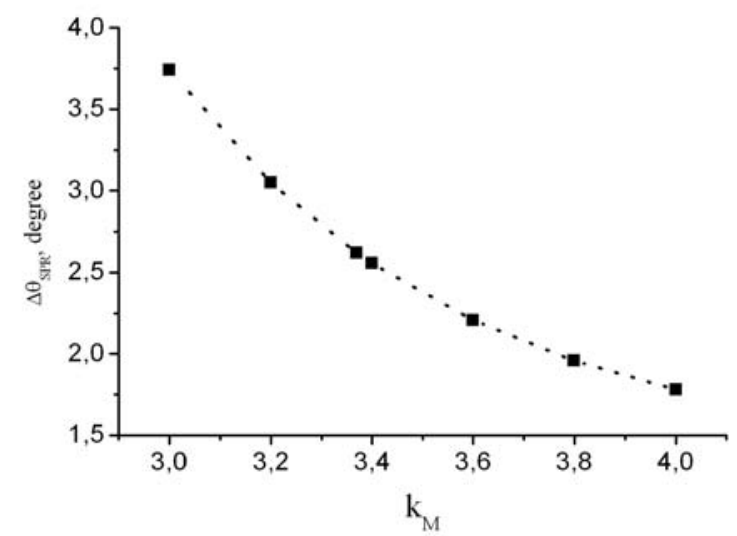

Fig. 6. Theoretical dependence of a shift of the resonance angle $\Delta \theta_{S P R}$ on the imaginary part of the dielectric permeability (coefficient of extinction of a metal film $k_{M}$ ).

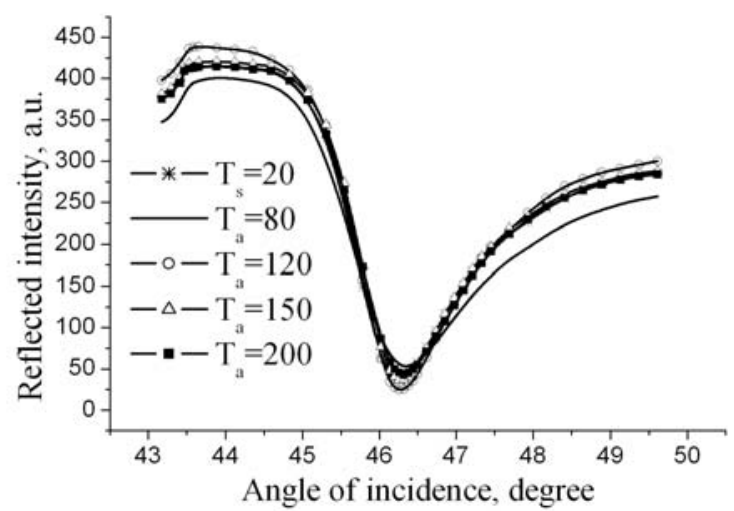

Fig. 7. Resonance curves (air) for a thin-film structure $\mathrm{Cr} / \mathrm{Au}$ obtained at the substrate temperature $T_{S}=20^{\circ} \mathrm{C}$ and annealed in the temperature interval $T_{A}=80 \div 200^{\circ} \mathrm{C}$. 
Table 1. Optical constants of the thin-film structure $\mathrm{Cr} / \mathrm{Au}$ obtained at the temperature $T_{S}=20^{\circ} \mathrm{C}$ and modified by the thermal annealing in the temperature interval $T_{A}=$ $80 \div 200^{\circ} \mathrm{C}$.

\begin{tabular}{|c|c|c|c|c|}
\hline $\begin{array}{c}\text { Temperature, } \\
{ }^{\circ} \mathrm{C}\end{array}$ & $n$ & $k$ & $d_{S P R}$ & $d_{A F M}$ \\
\hline 20 & 0.167 & 3.429 & 40.24 & 39.6 \\
\hline 80 & 0.178 & 3.422 & 36.993 & 38.4 \\
\hline 120 & 0.179 & 3.390 & 41.259 & 42.8 \\
\hline 150 & 0.154 & 3.370 & 41.752 & 44.4 \\
\hline 200 & 0.167 & 3.396 & 39.417 & 40.0 \\
\hline
\end{tabular}

The experimental study of optical characteristics of the thin-film structure $\mathrm{Cr} / \mathrm{Au}$ varying under the action of the low-temperature annealing was performed on the basis of measurements of the SPR curves and the integral scattering of light. The resonance curves were obtained in air and are presented in Fig. 7. The optical constants of the thin-film structure $\mathrm{Cr} / \mathrm{Au}$ obtained at the temperature $T_{S}=20^{\circ} \mathrm{C}$ and modified by the thermal annealing in the temperature interval $T_{A}=80 \div 200^{\circ} \mathrm{C}$ were calculated by the method of fitting of the theoretical SPR curves to the experimental ones within the one-layer model and are given in Table 1.

It is seen from Table 1 that the thicknesses of films obtained as a result of the numerical modeling of the experimental SPR curves $\left(d_{S P R}\right)$ and those found with the help of AFM $\left(d_{S P R}\right)$ correlate with one another. With regard for a small value of discrepancy, this result testifies to the adequacy of the chosen optical model of a gold coating and the correctness of the results. The minimum values of the coefficient of extinction correspond to the temperature interval of thermal annealing $(120 \div 150)^{\circ} \mathrm{C}$.

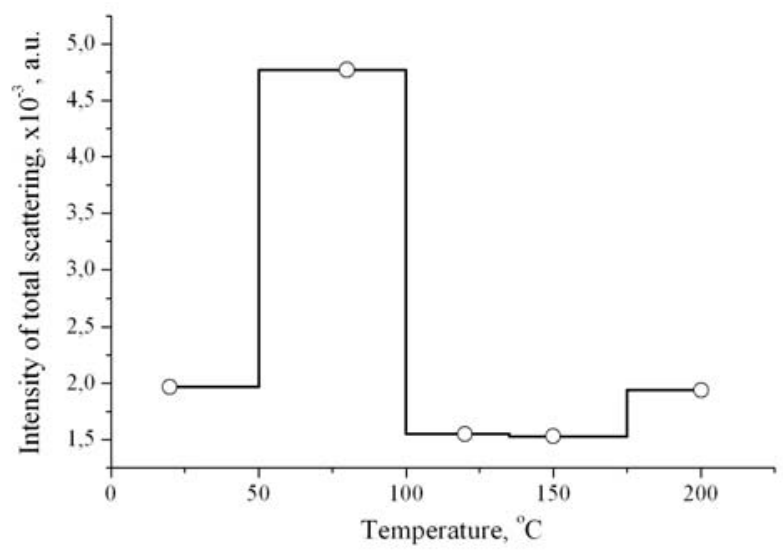

Fig. 8. Integral scattering at the normal incidence of light on thin-film structures $\mathrm{Cr} / \mathrm{Au}$ obtained at the substrate temperature $20^{\circ} \mathrm{C}$ and annealed in the temperature interval $80 \div 200^{\circ} \mathrm{C}$ under the excitation of SPR.

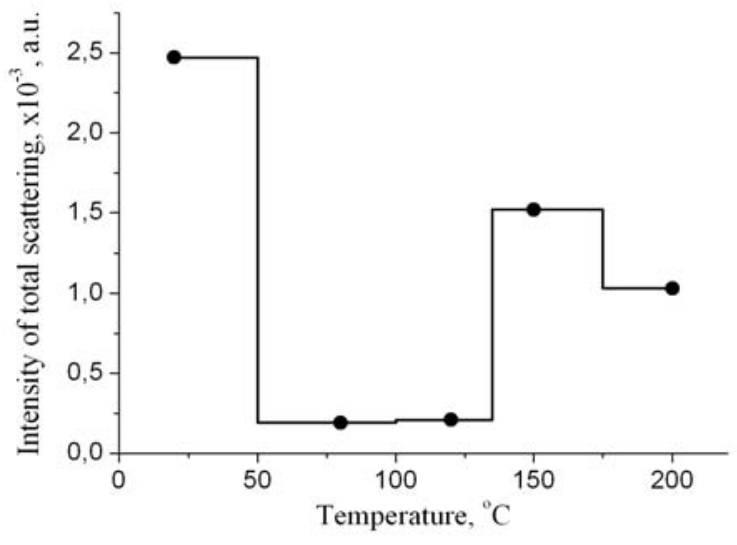

Fig. 9. Integral scattering at the normal incidence of light on gold films produced at the substrate temperature $20^{\circ} \mathrm{C}$ and annealed in the temperature interval $80 \div 200^{\circ} \mathrm{C}$.

Based on the results presented in Fig. 7 and Table 1, we can make the following concludions. On the whole, the SPR curves are similar to one another. The shape of a resonance curve is determined by peculiarities of the surface structure of metal films and depends on the ratio of the optical constants $n_{M}$ and $k_{M}$. At the same time, the minimum values of reflection at the minimum of the resonance $R_{\text {min }}$, the half-width of the resonance curve $\Delta R$, and the maximum reflection on the shoulders of a resonance curve, which are characteristic of the specimens annealed at $120^{\circ} \mathrm{C}$, ensure the best service characteristics of SPR-based transducers.

The scattering of light in the film bulk and on the interfaces can increase the contribution of the reflection signal not related to the formation of polariton states. The data on the integral scattering of light under conditions of the excitation of SPR (Fig. 8) testify to the minimum energy loss also in the specimens annealed at a temperature of about $120^{\circ} \mathrm{C}$. The comparison of the data on the integral scattering of light (Fig. 9 and Table 1) demonstrates the following qualitative correlation: the scattering decreases with the coefficient of extinction of a metal (imaginary part of the dielectric permeability). In this case, there is no correlation between the values of mean square roughness calculated from the data on the scattering of light and determined by the images of AFM. This allows us to assume that the scattering of light is determined by variations of the dielectric characteristics of a thin layer, rather than by topographical peculiarities of the surfaces of metal films.

\subsection{Service characteristics of transducers on the basis of SPR with thermal modification of a metal layer}

To forecast the stable operation of a sensor, it is necessary to study the long-term stability of the SPR curves, namely, the kinetics of temporal changes in the value of resonance angle $\left(\theta_{S P R}\right)$, which determines the base line of a device. 
As was shown above, the process of transformation of the surfaces of thin-film structures $\mathrm{Cr} / \mathrm{Au}$ subjected to the annealing includes two particular points. At an annealing temperature of $120^{\circ} \mathrm{C}$, the surface of crystallites is smoothed due to the disappearance of small-scale fracture faces. At $150^{\circ} \mathrm{C}$, we have registered a phase transition in the system, which results in an increase of the size of crystallites and in the smoothing of the whole surface relief. The values of a resonance angle, which were measured during 100 days at room temperature in air for thin-film structures $\mathrm{Cr} / \mathrm{Au}$ annealed at temperatures of 120 and $150^{\circ} \mathrm{C}$, are given in Table 2.

The presented data imply that the value of $\theta_{S P R}$ varies during the whole time of studies. The temporal instability of the films under study during three first days after the fabrication is related, apparently, to the relaxation of residual mechanical stresses. Then the stability is conserved during five days. At the same time, the character of relaxation identical for all specimens in subsequent days can be related to the adsorption of molecules on the film surface from the gas phase. Hence, the additional measures on the stabilization of $\theta_{S P R}$ are necessary even if the sensor operates in air. Moreover, the gold films under study reveal the tendency to a variation in physico-chemical characteristics during the long-term operation in a fluid medium, despite the sufficiently high chemical inertness of massive gold. To explain the changes in the optical parameters of thin films, it is necessary to consider not only changes in parameters of the upper layer, but also the possibility for aqueous solutions to enter the intercrystalline space of films with a polycrystalline structure. In this case, in view of a small thickness of films and the technological difficulties of the production of coatings without pores, the variations of only the conditions of thermal sputtering and annealing are not sufficient for the fabrication of sensitive SPR elements reliably functioning in aqueous medium. To prevent their "ageing," it is necessary to apply the transient layers [31] able to protect the surface and to stabilize the parameters of a transducer. Moreover, the thickness of a transient layer should be much less than the depth of penetration of a surface plasmon wave into the environment (100-200 $\mathrm{nm}$ for the emission in the visible part of the spectrum).

As the protective layer of a sensitive SPR element, the ordered self-assembled monolayers (SAMs) of molecules with controlled composition, structure, and thickness are used [32]. The layers on the basis of functionalized mercaptans (in particular, aliphatic thiols $\left.\left(\mathrm{HS}-\left(\mathrm{CH}_{2}\right)_{\mathrm{n}}-\mathrm{X}\right)\right)$ are most widely applied due to a simple production technology (by the method of long-term spontaneous chemosorption from a solution), stability, and functional variety. The close-packed molecular coating ensures the spatial shielding of a metallic surface by tens of angströms (C-H bond length is $~ 1.1 \AA$ ), which has no essential influence on the sensitivity of a sensor, and the terminal groups of thiol determine its physico-chemical and functional properties.
Table 2. Temporal variation of the resonance angle $\left(\theta_{S P R}\right)$ for thin-film structures $\mathrm{Cr} / \mathrm{Au}$ without annealing and subjected to the thermal annealing at 120 and $150^{\circ} \mathrm{C}$.

\begin{tabular}{|l|c|c|c|c|c|}
\hline \multirow{2}{*}{$\begin{array}{l}\text { Characteristic } \\
\text { of measured } \\
\text { specimens }\end{array}$} & \multicolumn{5}{|c|}{ Resonance angle (angular degrees) } \\
\cline { 2 - 6 } & $\begin{array}{c}\text { 1-st } \\
\text { day }\end{array}$ & $\begin{array}{c}\text { 3-rd } \\
\text { day }\end{array}$ & $\begin{array}{c}7 \text {-th } \\
\text { day }\end{array}$ & $\begin{array}{c}30 \text {-th } \\
\text { day }\end{array}$ & $\begin{array}{c}100-\text { th } \\
\text { day }\end{array}$ \\
\hline $\begin{array}{l}\text { Without } \\
\text { annealing }\end{array}$ & 45.96 & 46.08 & 46.08 & 46.14 & 46.26 \\
\hline $\begin{array}{l}\text { Annealing } \\
\text { temperature of } \\
120^{\circ} \mathrm{C}\end{array}$ & 46.08 & 46.14 & 46.14 & 46.26 & 46.32 \\
\hline $\begin{array}{l}\text { Annealing } \\
\text { temperature of } \\
150^{\circ} \mathrm{C}\end{array}$ & 46.02 & 46.08 & 46.08 & 46.14 & 46.20 \\
\hline
\end{tabular}

The optimization of stabilizing properties of a sensitive SPR-based element was implemented on the basis of measurements of the contact angle under the wetting with water, studies of resonance curves (Fig. 10), and the study of the kinetics of time variation of $\theta_{S P R}$ (Table 3) for gold films produced by the thermal evaporation in vacuum without heating with the subsequent low-temperature annealing at 120 and $150^{\circ} \mathrm{C}$ and modified by dodecanethiol $\left(\mathrm{HS}\left(\mathrm{CH}_{2}\right)_{11} \mathrm{CH}_{3}\right.$ is a hydrophobic coating, $1 \times 10^{-3}$ Mole/1 solution in ethyl alcohol, for $24 \mathrm{~h}$ at $297^{\circ} \mathrm{K}$ ).

It is seen from Table 3 that the resonance angle is invariable in the course of time only in the case where polycrystalline gold films were subjected to the thermal annealing at $T \sim 120^{\circ} \mathrm{C}$. Hence, the formation of a highly ordered defectless monolayer of gold thiolate and the full stabilization of characteristics of a transducer occur only on such substrates. Figure 10 indicates that the SPR curves for an as-prepared film of gold differ nonessentially from one another, which testifies to the absence of the formation of a self-organized monolayer. The measurements of the contact angle confirm this result. At the same time, we observe a shift for the film annealed at $120^{\circ} \mathrm{C}$, whose value is in good agreement with the formation of a surface coating monolayer [33].

Table 3. Temporal changes in the value of resonance angle $\left(\theta_{\mathrm{SPR}}\right)$ for gold films modified by dodecanethiol

\begin{tabular}{|l|c|c|c|c|c|}
\hline \multirow{2}{*}{$\begin{array}{l}\text { Characteristic } \\
\text { of measured } \\
\text { specimens }\end{array}$} & \multicolumn{5}{|c|}{ Resonance angle (angular degrees) } \\
\cline { 2 - 6 } & $\begin{array}{c}\text { 1-st } \\
\text { day }\end{array}$ & $\begin{array}{c}\text { 3-rd } \\
\text { day }\end{array}$ & $\begin{array}{c}\text { 7-th } \\
\text { day }\end{array}$ & $\begin{array}{c}30 \text {-st } \\
\text { day }\end{array}$ & $\begin{array}{c}\text { 100-st } \\
\text { day }\end{array}$ \\
\hline $\begin{array}{l}\text { Without } \\
\text { annealing }\end{array}$ & 44.12 & 44.17 & 44.21 & 44.21 & 44.17 \\
\hline $\begin{array}{l}\text { Annealing } \\
\text { temperature of } \\
120^{\circ} \mathrm{C}\end{array}$ & 44.17 & 44.17 & 44.17 & 44.17 & 44.17 \\
\hline $\begin{array}{l}\text { Annealing } \\
\text { temperature of } \\
150^{\circ} \mathrm{C}\end{array}$ & 44.26 & 44.17 & 44.21 & 44.26 & 44.21 \\
\hline
\end{tabular}




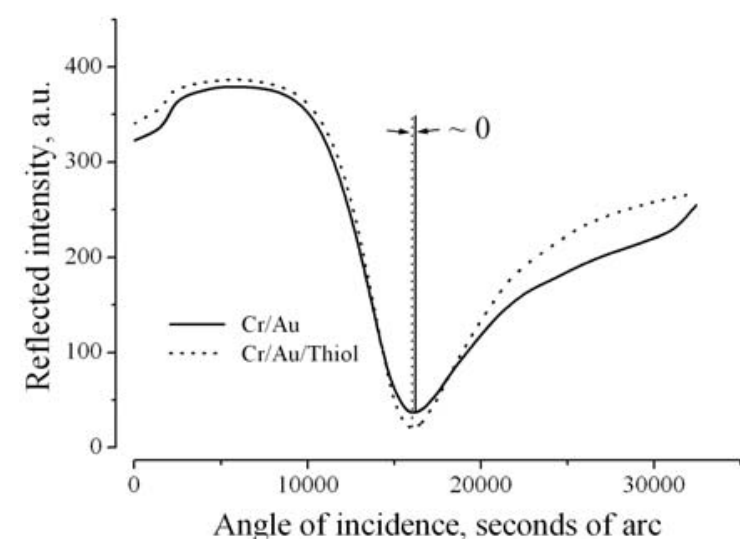

$a$

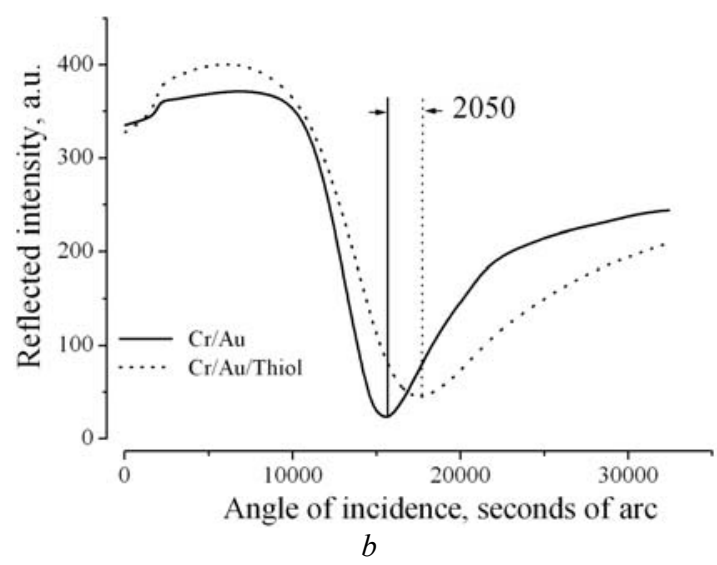

Fig. 10. SPR curves for as-prepaired (a) and annealed at $120^{\circ} \mathrm{C}$ (b) gold films modified by dodecanethiol.

We note that such specimens are characterized by the absence of a low-scale roughness of the surface. Therefore, the gold substrate containing the small-scale fractal structures comparable with the size of a thiol molecule $(\sim 1.7 \mathrm{~nm}$ for dodecanethiol) can serve as the probable reason for the formation of an imperfect coating. Unfortunately, the method of SPR allows one to obtain only the effective values of optical constants (real and imaginary parts of the dielectric permeability) and the mean thickness of a layer adsorbed on the surface of gold. At the same time, the method based on the analysis of the angular dependence of scattered light gives information about the correspondence of the effective thickness of an organic layer to variations of the relief height for the surface of gold [34]. The essence of the procedure consists in the calculation of the PSD functions within two mutually supplementing models, which involve the scattering of light by irregularities of the surface relief and by those of the dielectric permeability of the near-surface layer [35]. The qualitative coincidence of the functional PSD dependences on the spatial frequency for two wavelengths (441 and $632 \mathrm{~nm}$ ) allows us to conclude about the correlation in antiphase between the distribution of thicknesses of the thiol coating and the relief height of the surface of gold [36]. In the region of the tops of crystallites, the formation of an organic monolayer occurs analogously to the classical ideas of smooth monocrystalline surfaces. On the concave parts of the surface (region of dents), we can observe the distortions of the dense hexagonal structure of gold thiolate, which is favored by the steric limitaions and the accumulation of fine defects in the thin gold films in the domains of intercrystalline boundaries commensurable with the size of a dodecanethiol molecule. Therefore, the thickness of a dielectric layer of thiol in such domains can significantly exceed the size characteristic of a monolayer coating due to the overlapping of aliphatic chains.

\section{Conclusion}

We have carried out the complex analysis of variations in the structural (low-angle diffraction of X-ray emission, atomic-force microscopy) and optical (shape of the SPR curve, long-term stability of the resonance, optical constants and integral scattering of light) characteristics of gold films under the influence of a low-temperature annealing in the interval $(80 \div 200)^{\circ} \mathrm{C}$ in order to improve the efficiency of SPR transducers and the stability of their parameters at a storage.

It is shown that the best service characteristics of SPR-based transducers on the basis of polycrystalline films of gold produced by the method of thermal evaporation in vacuum (VUP-4, a residual vapor pressure of $4 \cdot 10^{-4} \mathrm{~Pa}$, a sputtering rate of $40-50 \AA / \mathrm{s}$ ) with the use of an adhesive chromium sublayer (thickness of about $1 \mathrm{~nm}$ ) on quartz substrates without heating are attained with the help of the subsequent annealing $\left(120^{\circ} \mathrm{C}\right)$ for $30 \mathrm{~min}$ in air. At this temperature, we observed the smoothing of a small-scale relief of the gold film surface, which allows us to decrease the light scattering intensity under conditions of SPR, to decrease the coefficient of extinction, to stabilize the optical characteristics, and to form the defectless nano-sized protective stabilizing layers on the surface.

\section{Acknowledgements}

This research was performed in the framework of state project No. 0112U002349 "Physical and technological aspects of development of modern semiconductor materials and functional structures for nano- and optoelectronics", approved Nov. 28, 2011, supported by National Academy of Sciences of Ukraine.

\section{References}

1. A. Brecht, G. Gauglitz, Optical probes and transducers // Biosensors and Bioelectronics, 10, p. 923-936 (1995). 
2. J.J. Ramsden, Optical biosensors // Journal of molecular recognition, 10, p. 109-120 (1997).

3. J. Davies, Surface plasmon resonance - the technique and its applications to biomaterial processes // Nanobiology, 3, p. 5-16 (1994).

4. Surface Plasmon Resonance Based Sensors (Springer Series on Chemical Sensors and Biosensors) / Ed. by J. Homola. - Berlin-HeidelbergNew York: Springer-Verlag, 2006. - 251 p.

5. D.R. Shankaran, K.V. Gobi, N. Miura, Recent advancement in surface plasmon resonance immunosensors for detection of small molecules of biomedical, food and environmental interest // Sensors and Actuators B: Chemical, 121(1), p. 158-177 (2007).

6. X.D. Hoa, A.G. Kirk, M. Tabrizian, Towards integrated and sensitive surface plasmon resonance biosensors: A review of recent progress // Biosensors and Bioelectronics, 23, p.151-160 (2007).

7. P. Singh, Biosensors: Historical Perspectives and Current Challenges // Sensors and Actuators B, 229, p. 110-130 (2016).

8. J. Zhang, L. Zhang, W. Xu, Surface plasmon polaritons: physics and applications // Journal of Physics D: Applied Physics, 45(11), p. 113001 (2012).

9. E. Kretschmann, Determination of optical constants of metals through the stimulation of surface plasmon oscillations // Z. Phys, 241, p.313-324 (1971).

10. A. Otto, Excitation of nonradiative surface plasma waves in silver by the method of frustrated total reflection // Zeitschrift fur Physik a Hadrons and Nuclei, 216(4), p.398-410 (1968).

11. Handbook of Surface Plasmon Resonance / Edited by R.B.M. Schasfoort and Anna J. Tudos, Cambridge (UK): Royal Society of Chemistry, 2008, 426 p.

12. H.E. de Bruijn, R.P.H. Kooyman, J. Greve, Choice of metal and wavelength for surface-plasmon resonance sensors: some considerations // Applied Optics, 31(4), p. 440-442 (1992).

13. E. Fontana, Thickness optimization of metal films for the development of surface-plasmon-based sensors for nonabsorbing media // Applied Optics, 45(29), p. $7632-7642$ (2006).

14. Yu.M. Shirshov, A.V. Samoylov, S.A. Zinyo, E.R. Surovceva, V. Mirskiy, Bimetallic layers increase sensitivity of affinity sensors based on surface plasmon resonance // Sensors, 2, p.62-70 (2002).

15. B.P. Benjamin, C. Weaver, The adgesion of evaporated metal films on glass // Proc. Roy. Soc. A., 261(7), p. 516-531 (1961).

16. M.J. Verkerk, I.J.M. Raaijmakers, Topographic characterization of vacuum-deposited films by optical methods // Thin Solid Films, 124, p. 271275 (1985).
17. F. Parmigiani, M. Scagliotti, G. Samoggia, G.P. Ferraris, Influence of the growth conditions on the optical properties of thin gold films // Thin Solid Films, 125, p. 229-234 (1985).

18. A.J. Braundmeier, E.T. Arakawa, Effect of surface roughness on surface plasmon resonance adsorption // Journal Physics Chemistry Solids, 35, p. 517-520 (1974).

19. W.H. Weber, Modulated surface-plasmon resonance for in situ metal-film surface studies // Physical Review Letters, 39, p. 153-156 (1977).

20. S.I. Lysenko, B.A.Snopok, V.A. Sterligov, Scattering of surface plasmons and normal waves by thin gold films // Optics and Spectroscopy, 188(4), p.618-628 (2010).

21. L.H. Guo, J.S. Facci, G. McLendon, R. Mosher, Effect of gold topography and surface pretreatment on the self-assembly of alkanethiol monolayers // Langmuir, 10, p. 4588-4593 (1994).

22. D. Tanaka, S. Shinohara, E. Usukura, P. Wang, K. Okamoto, K. Tamada, High-sensitivity surface plasmon resonance sensors utilizing highrefractive-index silver nanoparticle sheets // Japanese Journal of Applied Physics, 53, p. 01AF01-01AF07 (2014).

23. S.J. Gregg, K.S.W. Sing, Adsorption, Surface Area and Porosity, London, New York: Academic Press, 1967. $-371 \mathrm{p}$.

24. O.V. Rengevich, Y.M. Shirshov, Y.V. Ushenin, A.G. Beketov, Separate determination of thickness and optical parameters by surface plasmon resonance: accuracy consideration // Semiconductor physics, quantum electronics and optoelectronics, 2(2), p. 28-35 (1999).

25. R.M.A. Azzam, N.M. Bashara, Ellipsometry and Polarized Light, Amsterdam, New York, Oxford: North-Holland publishing company, 1977. - 529 p.

26. B.A. Snopok, K.V. Kostyukevich, S.I. Lysenko, P.M. Lytvyn, P.E. Shepeliavii, O.S. Lytvyn, S.V. Mamykin, S.A. Zynio, S.A. Kostyukevich, E.F. Venger, Yu.M. Shirshov, Optical biosensors based on the surface plasmon resonance phenolmenon: optimization of the metal layer parameters // Semiconductor Physics, Quantum Electronics and Optoelectronics, 4(1), p. 56-69 (2001).

27. P.E. Strizhak, B.A. Snopok, T. Melnik, E.V. Kostyukevich, S.I. Lysenko, P.E. Shepeliavii, S.L. Priatkin, S.A. Kostyukevich, Yu.M. Shirshov, E.F. Venger, Multifractal properties of polycrystalline gold films, International Conference on Correlation Optics/ Angelsky O.V., Editor // Proceedings of SPIE, 3904, p.374-385 (1999).

28. A. Duparre, Light scattering of thin dielectric films, in: Handbook of optical properties / Ed. by R.E. Hummel, K.H. Guenther, CRC Press, v.1, p. 123-187 (1995).

29. J.M. Elson, Theory of light scattering from a rough surface with an anhomogeneous dielectric 
permittivity // Physical Review B, 30, p. 5460-5480 (1984).

30. C. Ruppe, A. Duparre, Roughness analysis of optical films and substrates by atomic force microscopy // Thin Solid Films, 288, p. 8-13 (1996).

31. E. Delamarch, B. Michel, H.A. Biebuyck, C. Gerber, Golden Interfaces: The Surface of SelfAssembled Monolayers // Advanced Materials, 8, p. 719-729 (1996).

32. A. Ulman, Formation and structure of selfassembled monolayers // Chem. Rev., 96, p. 15331554 (1996).

33. H. Keller, W. Schrepp, H. Fuchs, Self-assembled organic films on gold and silver // Thin solid films, 210/211, p. 799-802 (1992).
34. C. Amra, From light scattering to the microstructure of thin-film multilayers // Applied Optics, 32, p. 5481-5491 (1993).

35. R. Silvennoinen, J. Rasanen, M. Savolainen, K.E. Peiponen, J. Uozumi, T. Asakura, On simultaneous optical sensing of local curvature and roughness of metal surfaces // Sensors and Actuators A, 51, p. 117-123 (1996).

36. S.I. Lysenko, B.A. Snopok, E.V. Kostyukevich, S.A. Zinio, V.A Sterligov., Yu.M. Shirshov, E.F. Venger, Light scattering of thin dielectric films: self-assembled monolayers on the surface of polycrystalline gold, International Conference on Correlation Optics / Angelsky O.V., Editor. // Proceedings of SPIE, 3904, p. 476-487 (1999). 